\title{
PERCEPCIÓN LOCAL DEL ESTADO AMBIENTAL EN LA CUENCA BAJA DEL RÍO MANZANARES
}

\author{
Ángela María Plata ${ }^{1}$ \\ Danny Ibarra Vega ${ }^{2}$ \\ Universidad Sergio Arboleda
}

Recibido el 1 de diciembre de 2014, aprobado el 27 de abril de 2015 y actualizado el 09 de noviembre de 2015

DOI: 10.17151/luaz.2016.42.15

\section{RESUMEN}

Los problemas ambientales que afectan a las cuencas hidrográficas en Colombia tienen múltiples causas, una de ellas es la ausencia de una cultura ambiental en las comunidades que viven o frecuentan zonas aledañas a las corrientes hídricas. El principal objetivo de este trabajo era recopilar la percepción local sobre el estado ambiental de la cuenca baja del río Manzanares, ubicada en el departamento del Magdalena e incentivar a los pobladores a reflexionar sobre su papel frente a la solución de las principales problemáticas. Para lograr este objetivo se utilizaron diferentes herramientas metodológicas, 1) Encuesta dirigida a los pobladores de la zona, 2) talleres participativos con estudiantes, 3) desarrollo de recorridos de reconocimiento ambiental y territorial. A través de estos últimos se recopiló la percepción de los participantes sobre componentes ambientales básicos: agua, aire, suelo, flora, fauna y saneamiento básico. Se realizaron dos recorridos con grupos diferentes, teniendo un total 50 estudiantes del grado décimo de un colegio ubicado en la zona de estudio y algunos estudiantes universitarios. A través de este estudio se encuentra que la principal problemática percibida por la comunidad de la cuenca baja del río Manzanares en Santa Marta, es la contaminación del agua por el aporte de aguas residuales y la disposición no controlada de residuos sólidos; esta última causa, ligada a la falta de una cultura ambiental no solo en los residentes de la ciudad sino también en sus visitantes.

\section{PALABRAS CLAVE}

Percepción, recorrido ambiental, cuenca hidrográfica.

\section{LOCAL PERCEPTION OF THE ENVIRONMENTAL STATUS IN THE LOWER BASIN OF THE MANZANARES RIVER}

\begin{abstract}
Environmental problems affecting hydrographic basins in Colombia are generated by multiple factors, being one of them the lack of environmental culture in local communities living in adjacent areas to the water currents. The main objective of this work was to compile the local community perception about the environmental status of the lower basin of the Manzanares River, located in the department of Magdalena, and to encourage settlers to reflect on their role for the solution of the major problems. In order to achieve this objective, different methodological tools were used: 1) A survey with local people, 2) participatory workshops with students, and 3) environmental and territorial recognition routes. By using these tools, it was possible to collect the perception of the participants
\end{abstract}


about basic environmental components: water, air, soil, flora, fauna and basic sanitation. Two routes were done with different groups and a total of 50 tenth grade students from a school in the area and some university students. This study found that the main environmental problem that the community of the lower basin of the Manzanares river in Santa Marta perceives is water pollution generated by the supply of wastewater and by the uncontrolled disposal of solid wastes; this last cause is linked to the lack of environmental culture, not only in the city residents but also in its visitors.

KEY WORDS: Perception, environmental route, hydrographic basin.

\section{INTRODUCCIÓN}

Actualmente más de la mitad de la población mundial vive en las ciudades (Dye, 2008), y se espera que para el año 2050 este fenómeno aumente a más de las dos terceras partes (United Nations, 2010). De esta manera, podemos ver que el crecimiento de la población urbana cada vez altera más los ecosistemas inmersos en los centros urbanos; siendo los ríos uno de los principales ecosistemas presentes en ellos.

Las cuencas hidrográficas y sus áreas de inundación proporcionan tierra fértil para la agricultura, agua para consumo y riego y un medio para transportar bienes o en algunos casos indebidos para disponer residuos (Vollmer y Grêt-Regamey, 2013). La mayoría de las ciudades han llegado a depender de los servicios hidrológicos de las cuencas o partes de las cuencas, así cierta infraestructura urbana puede haber sido pensada con el fin de aprovechar de manera eficiente los servicios que brindan estos cuerpos de agua (Lee, 2006; Gómez-Baggethun y Barton, 2013). Con el rápido desarrollo de la economía de las ciudades, los efectos de las actividades antropogénicas sobre los ecosistemas fluviales están aumentando y la contaminación por fuentes no puntuales es cada vez más problemática (Wang et al., 2014, p. 1233).

Una de las formas para mitigar los problemas ambientales es generar una transformación cultural en la población, para lo cual se plantea el uso de la educación ambiental como herramienta básica. Esta tiene como principal misión la formación de un vínculo entre los valores, conocimientos, habilidades, para permitir a los individuos la unión al entorno al que pertenecen (Vargas et al., 2013, p. 156).

"Dentro de la educación ambiental se encuentra la educación ambiental comunitaria, que es un campo abierto al pensamiento y a la acción constructiva, en donde los resultados pueden convertirse gradualmente en propuestas creativas para un futuro diferente" (Tréllez, 2002, p. 8).

A pesar de que la transformación cultural en una comunidad es un proceso que va más allá de la educación ambiental e implica un proceso permanente y constante, es necesario impulsar estos espacios, para incentivar la protección de nuestros ecosistemas desde iniciativas comunitarias.

Por tanto, se evidencia la necesidad de conocer la percepción de las comunidades locales acerca del estado ambiental de su entorno, 
reconociendo las principales problemáticas, las causas y consecuencias que estas conllevan. Múltiples autores han resaltado la importancia de abordar la percepción de la gente en torno a temas ambientales. Autores como Gómez, Gutiérrez y Montes (2013, p. 30), Delgado, Trujillo y Torres (2013) y Patiño y Tobasura (2011), incorporan como componente fundamental en sus trabajos la percepción del ser humano en el análisis de problemáticas ambientales y en el proceso para plantear alternativas de solución frente a estas.

El presente estudio fue desarrollado en la cuenca baja del río Manzanares, puntualmente en la ciudad de Santa Marta, departamento del Magdalena, en la vertiente noroccidental de la Sierra Nevada de Santa Marta. La cuenca limita por el este con la cuenca del río Piedras, por el sur con la cuenca del río Gaira y por el oeste con el mar Caribe (Figura 1).

En esta zona se evidencia la ausencia de una cultura ambiental en la comunidad que vive en zonas aledañas al río; lo cual se refleja en el aporte constante de residuos sólidos y líquidos directamente al río, sin ningún tipo de tratamiento. De igual forma, se muestra una falta de sentido de pertenencia con el río, lo que ha ocasionado un detrimento permanente en la calidad de vida de la comunidad samaria, un daño a la estética paisajista, un fuerte impacto negativo en los ecosistemas asociados y un aumento en el riesgo sanitario de los habitantes de la zona.

Dado este panorama, el objetivo principal de este estudio es recopilar la percepción local del estado ambiental en la cuenca baja del río Manzanares, con el fin de proponer estrategias pedagógicas para su recuperación. De esta manera, se encontró que muchos de los habitantes tienen una percepción negativa sobre el río, la cual se refleja en los resultados de este estudio. La aproximación a la percepción de la comunidad se realizó a través de tres herramientas básicas: 1) encuestas dirigidas a los pobladores de la zona, 2) talleres participativos con estudiantes y 3 ) dos recorridos de reconocimiento ambiental y territorial a través de los cuales se recopiló la percepción de los participantes frente a componentes ambientales básicos: agua, aire, suelo, flora, fauna y saneamiento básico; en estos se contó con un total 50 estudiantes del grado décimo de un colegio ubicado en la zona de estudio y algunos estudiantes universitarios. De manera complementaria a esta última herramienta se utilizó la metodología de la cartografía social, a través de la cual se pudo analizar la relación del participante con su territorio y la forma en que este asocia las principales situaciones ambientales con la cuenca.

El uso de las herramientas anteriormente expuestas, además de servir para la recopilación de la información de la investigación, fue útil para sensibilizar a las personas de la comunidad participante, ya que esto las hace plantearse varios cuestionamientos, como su responsabilidad frente a los principales problemas ambientales en la cuenca y las iniciativas que ellas pueden tener para su recuperación. 


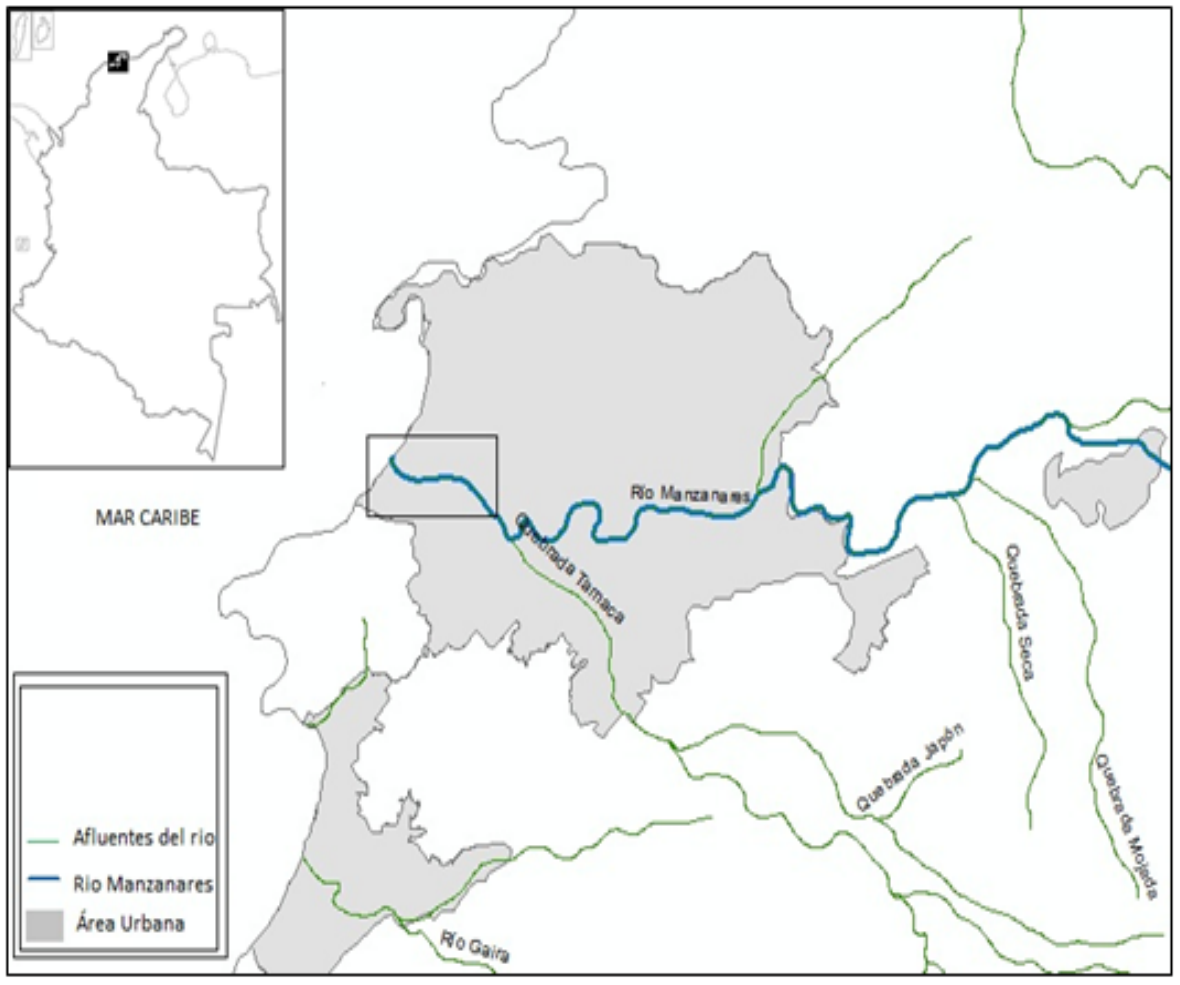

Fuente: los autores.

Figura 1. Mapa de ubicación del área de estudio en la cuenca baja del río Manzanares.

\section{MATERIALES Y MÉTODOS}

La metodología utilizada para el desarrollo del estudio fue cualitativa y participativa, enfocada a incentivar en la población local una posición crítica frente al estado ambiental actual de la cuenca baja del río Manzanares. Adicionalmente, se utilizó el método de la triangulación, utilizando como fuentes principales las siguientes herramientas: encuesta, taller participativo, y recorrido de reconocimiento ambiental y territorial.

Estas herramientas se describen a continuación:

1) Encuesta. El principal objetivo de la encuesta era reconocer la problemática del río a partir del conocimiento de la comunidad aledaña, de igual manera analizar cómo el estado actual de esta fuente afecta a quienes habitan cerca de ella.

La encuesta fue aplicada a 30 familias localizadas en la cuenca baja del río Manzanares dentro de la ciudad de Santa Marta. Gran parte de ellas viven en barrios afectados por las principales problemáticas del río, especialmente en el barrio Manzanares y en La Tenería. Los participantes eran personas de todas las edades, principalmente entre los 50 y 60 años de edad ( $27 \%$ de los encuestados) (ver Figura 2). 


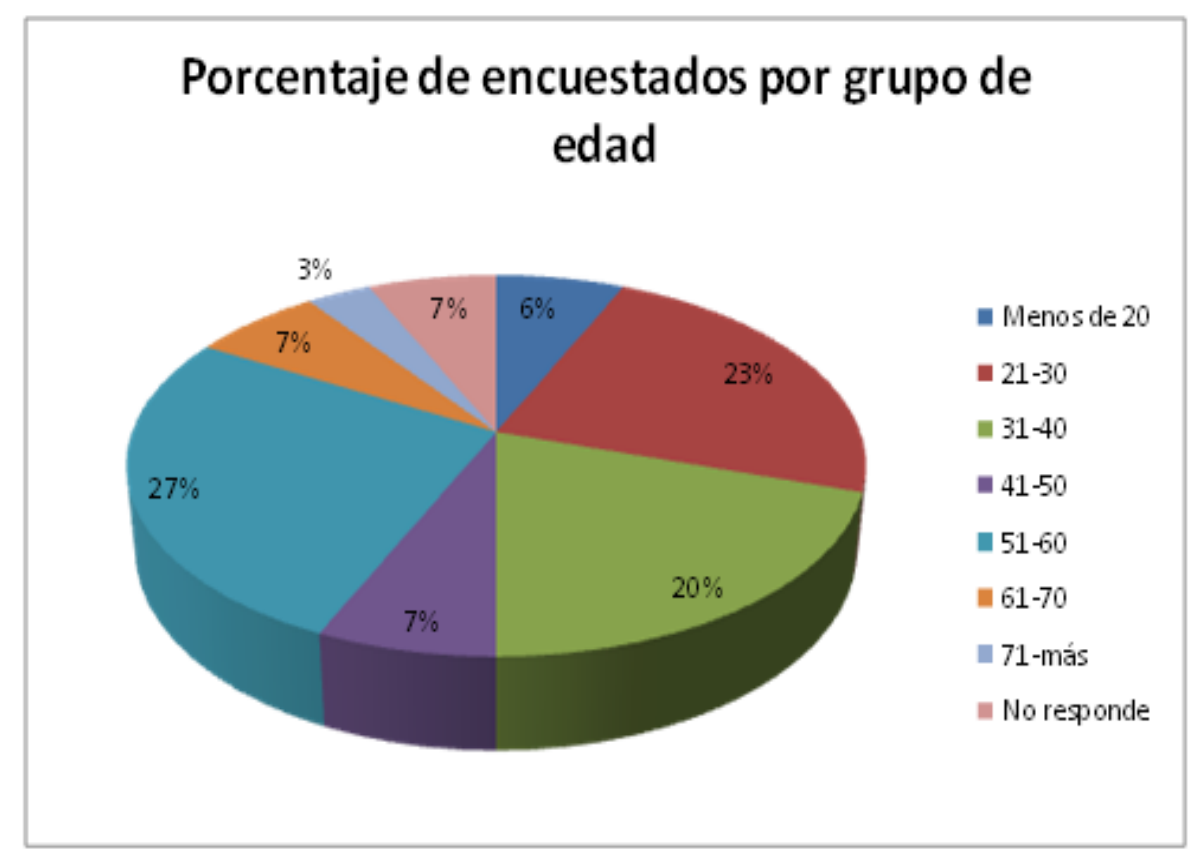

Figura 2. Porcentajes de personas encuestadas por grupos de edad.

2) Taller participativo. Este se realizó con docentes del colegio y estudiantes de grados 10 y 11 de la región. Los participantes se organizaron en grupos y a partir de una discusión procedieron a socializar su lluvia de ideas plasmada en tarjetas donde abordaban la siguiente pregunta: ¿Cómo aportar a la recuperación del río Manzanares?

3) Recorrido de reconocimiento ambiental y territorial. El objetivo de este taller era acercarse a la percepción de algunos estudiantes pobladores de la cuenca del río, acerca de las principales problemáticas presentes en la cuenca baja del río Manzanares. Los estudiantes con los que se hicieron los talleres, eran en su mayoría del colegio Agustín Nieto Caballero ubicado en el barrio Manzanares en la ciudad de Santa Marta, además se contó con la participación de algunos estudiantes de la Universidad del Magdalena. Este fue aplicado a dos grupos distintos.

Cada grupo recibió las instrucciones del taller y posteriormente realizó el recorrido por una zona previamente definida por el grupo de trabajo. En campo se realizaron las observaciones pertinentes $y$, posteriormente, en un aula de clase se procedió a realizar un ejercicio de cartografía social.

Para el desarrollo de este recorrido se utilizaron las siguientes herramientas:

a) Cuadernillo-cuestionario. Estos contenían preguntas direccionadas a componentes ambientales previamente definidos (Agua, Aire, Suelo, Flora, Fauna, Saneamiento Básico). Cada cuadernillo era asignado a un grupo diferente, constituido para abordar un componente específico.

b) Cartografía social. Cháves (2001) define la cartografía social como "el método para promover y facilitar los procesos de planeación participativa y de gestión social de las comunidades en el proceso de ordenamiento y desarrollo de sus territorios"(Mora y Jaramillo, 2003, p. 7); adicionalmente, Mora y Jaramillo (2003, p. 10) afirman que en la investigación de la 
cartografía social, la comunidad es partícipe de la investigación, aporta sus saberes y experiencias al tiempo que recibe de los demás; los autores consideran que los mapas se adecuan y favorecen la cultura de los narradores orales $y$, además, que la construcción colectiva de mapas permite la reactualización de la memoria individual y colectiva.

En la Figura 3 se muestran algunos participantes del recorrido observando una tubería que vierte aguas residuales en el río.

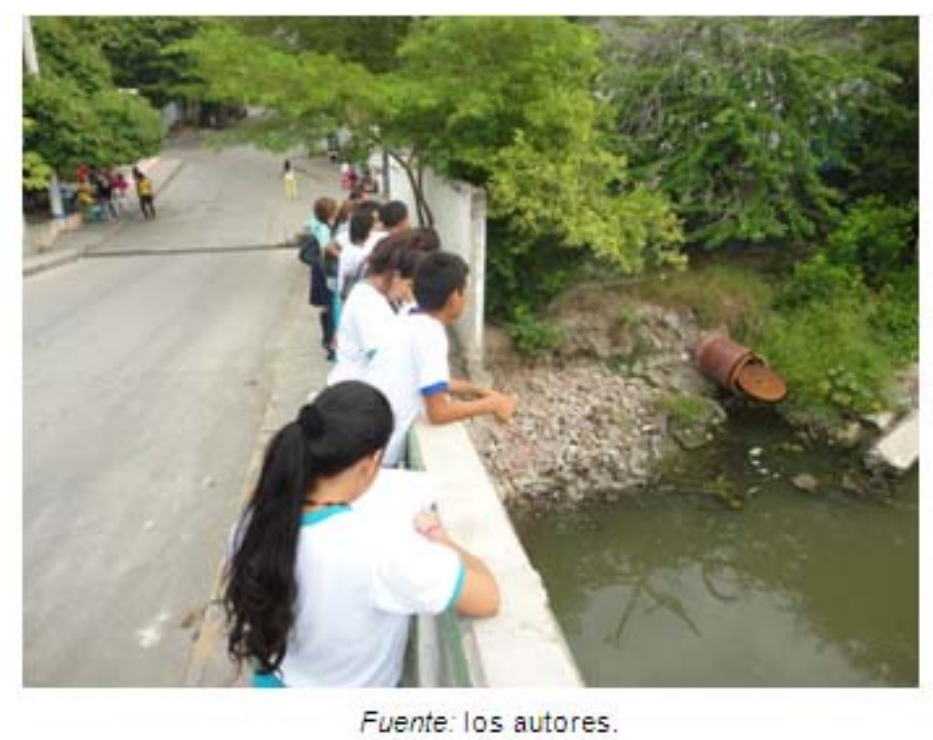

Figura 3. Estudiantes realizando observaciones durante el recorrido.

\section{RESULTADOS Y DISCUSIÓN}

\section{Resultados obtenidos a través del recorrido de reconocimiento ambiental y territorial}

\section{Recurso Agua}

Los participantes del taller reconocen que existen dos fuentes hídricas principales en su territorio, estas son el río Manzanares y el río Bonda. Para ellos, el estado actual del agua es muy malo, afirman que el agua del río Manzanares se encuentra muy contaminada por desechos arrojados.

Adicionalmente, indican que los principales problemas relacionados con el agua en su territorio son: estancamiento de agua debido a las barreras generadas por los desechos, contaminación del río por los distintos tipos de desechos, color del agua por la llegada de aguas negras y alcantarillas. Las principales causas de los problemas en su territorio son: arrojar basura al río, quema de basuras y arrastre de material resultante al agua, desechos tóxicos de industrias; por su parte, las principales consecuencias encontradas fueron: personas enfermas, muerte de fauna del río por los desechos, contaminación general del río Manzanares.

Durante el recorrido realizado, y por lo general, en la cuenca baja del río Manzanares se pueden observar diferentes tipos de residuos, entre ellos: llantas, colchones, restos de sofá, ropa, escombros, basura, animales 
muertos, entre otros. Estos residuos se generan principalmente en la comunidad, por las personas que viven en los alrededores del río y que no tienen conciencia de lo que hacen, y los vierten al río. Adicionalmente, se observó directamente el aporte de vertimientos que, de acuerdo con el conocimiento de los participantes, provienen de Metroagua, que es la empresa de servicios públicos que opera los sistemas de acueducto y alcantarillado sanitario en Santa Marta (ver Figura 2).

Todos estos problemas del recurso hídrico se relacionan con el manejo de los residuos sólidos y líquidos, por ello, la comunidad percibe que la solución de estos problemas se encuentra directamente ligada al manejo de estos. Por tanto, se plantea como posibles soluciones: evitar arrojar basura al río, hacer campañas para concientizar a la gente del daño que están causando y que se puede cambiar el estado del río.

Finalmente, los participantes reflexionan sobre el futuro del recurso en su región y plantean que en 10 años el río Manzanares estará muy acabado, ya que ahora las personas no tienen conciencia de lo que hacen y afirman que a futuro podría no existir esta fuente hídrica.

En la Figura 4, se refleja parte de la percepción de la comunidad plasmada en el ejercicio de cartografía social, en esta se reitera la relación que encuentra la comunidad entre el mal manejo de los residuos, el aporte de aguas residuales y la calidad del agua.

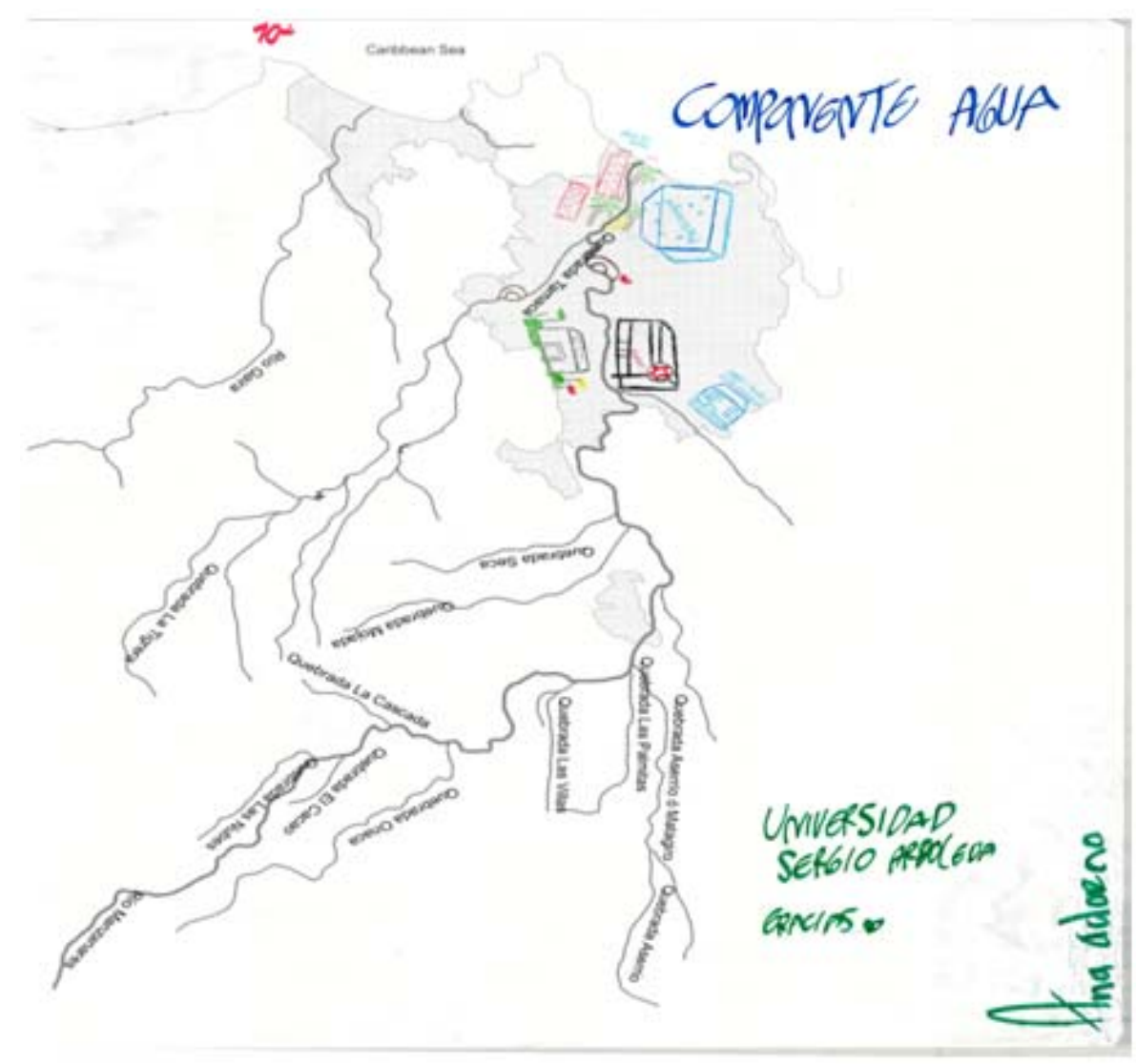

Figura 4. Imagen del taller de cartografía social, componente Agua. 


\section{Recurso Aire}

El estado actual del aire en la cuenca baja del río, desde la percepción de la comunidad, es regular. En el recorrido se percibió quema de basuras, cocinas informales con leña, emisiones de vehículos y motos, malos olores en el ambiente, entre otros.

Los principales problemas relacionados con este componente, son: contaminación del aire, descuido de los recursos naturales, tala de árboles, deficiencia en procesos de ordenamiento territorial. Las principales causas encontradas son la tala de árboles (percibida como problema y como causa, ya que la ausencia de vegetación disminuye el secuestro de carbono), falta de cobertura de gas domiciliario (lo cual lleva al uso de leña y carbón), mala organización urbana, la creencia de muchos pobladores de que los recursos son infinitos. Las consecuencias de estos problemas son el establecimiento de cocinas informales, erosión del suelo, establecimiento de basureros improvisados, emisiones a la atmósfera.

Las fuentes de contaminación del aire observadas en el recorrido, están relacionadas principalmente con hogares, empresas, vehículos, fábricas artesanales, personas con poco sentido común. Frente a estos problemas relacionados se plantea el uso de energías alternativas en vehículos, como gas natural o energía eléctrica (el caso de las motos eléctricas, de gran utilidad en ciudades intermedias); eliminar quema de basuras y cocinas de leña y, sobre todo, la enseñanza y educación enfocada al manejo de residuos y reciclaje, ya que un manejo adecuado de los residuos disminuye la posibilidad de la quema de estos, evitando emisiones impactantes en el ambiente y, por ende, en la salud humana. Los participantes creen que, contando con suerte, se desarrollarán productos y servicios que reducirán el impacto ambiental que se ha provocado, de no ser así se seguirá afectando el aire en la zona.

En la Figura 5 se puede apreciar la percepción que la comunidad tiene frente al componente Aire. En esta se muestran tres zonas principales, una donde el aire es fluido, hay presencia de árboles y campos abiertos, otra donde hay empresas, urbanizaciones y vehículos, y por ende contaminación del aire. Finalmente, una en la que predominan los malos olores, causados principalmente por quema de basura. 


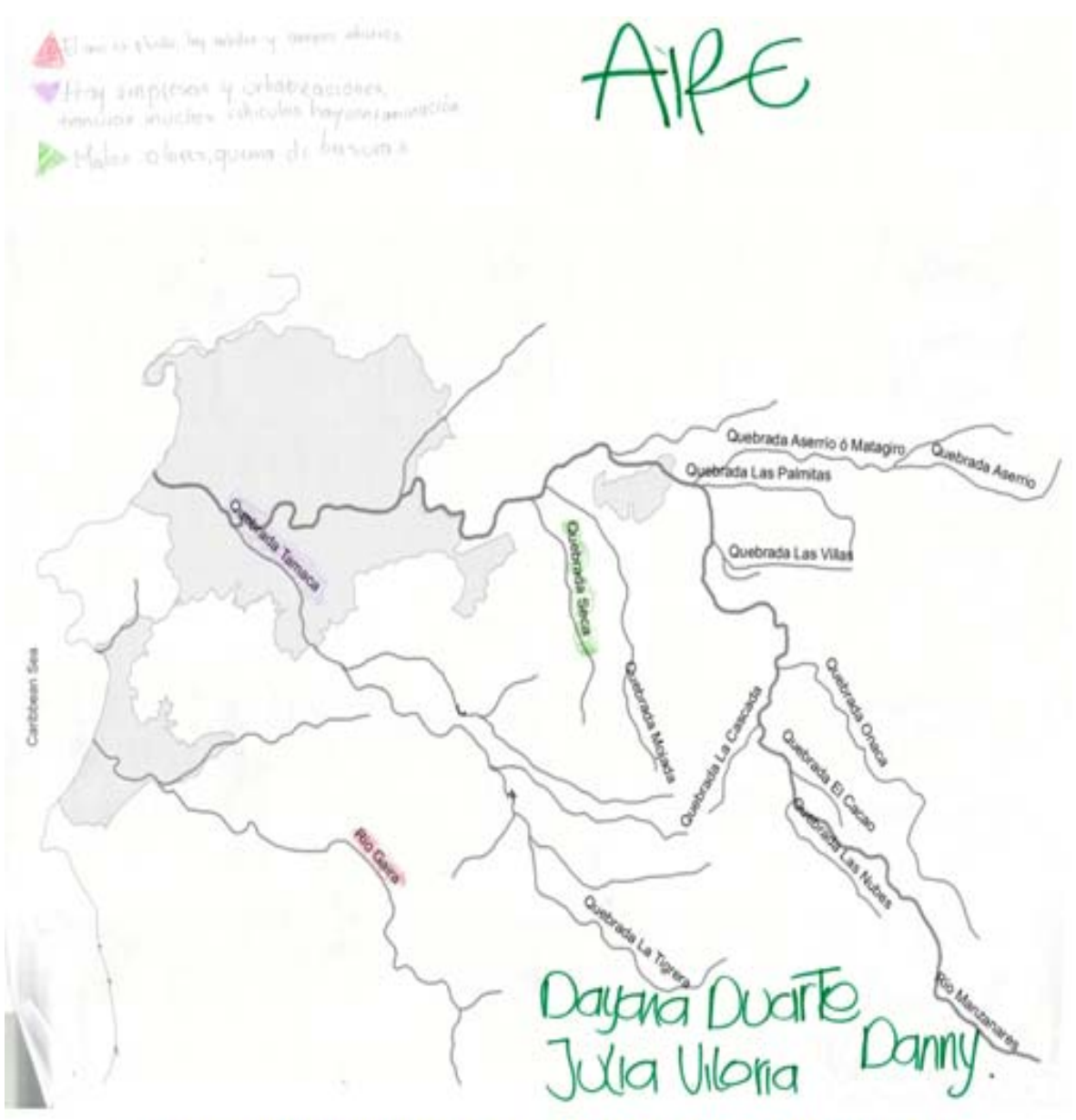

Figura 5. Imagen del taller de cartografía social, componente Aire.

\section{Recurso Suelo}

Se percibe que el principal uso que se le da al suelo en la cuenca baja del río es la construcción de vivienda; esto incrementa una de las principales problemáticas en la cuenca, las inundaciones, lo cual se da ya que gran parte de las casas se encuentran ubicadas sobre la ronda del río de manera ilegal. Adicionalmente a esto, se afirma que el suelo en el territorio está en mal estado, deteriorado por las basuras y escombros presentes en este. Estos dos problemas (basuras e invasión de viviendas), tienen como consecuencia la contaminación del río, que poco a poco se va deteriorando más.

Durante el recorrido, los participantes encontraron de manera visible una gran cantidad de residuos dispuestos en el suelo, como: bolsas plásticas, pañales desechables, muebles, vasos, basura en general, los cuales provienen principalmente de las viviendas aledañas, empresas y fábricas que arrojan sus residuos. Este problema visibilizado en el recorrido se puede prevenir evitando arrojar basuras y escombros en el territorio.

Frente al estado actual del suelo, se cree que este podría ser recuperado si las personas generan conciencia, o si no en 10 años estará más deteriorado. 
En la Figura 6, se reflejan las principales problemáticas relacionadas con el suelo, observadas por los participantes; en esta imagen se percibe y reitera como problemáticas principales: el aporte de residuos sólidos al suelo y la construcción sobre la ronda hídrica.

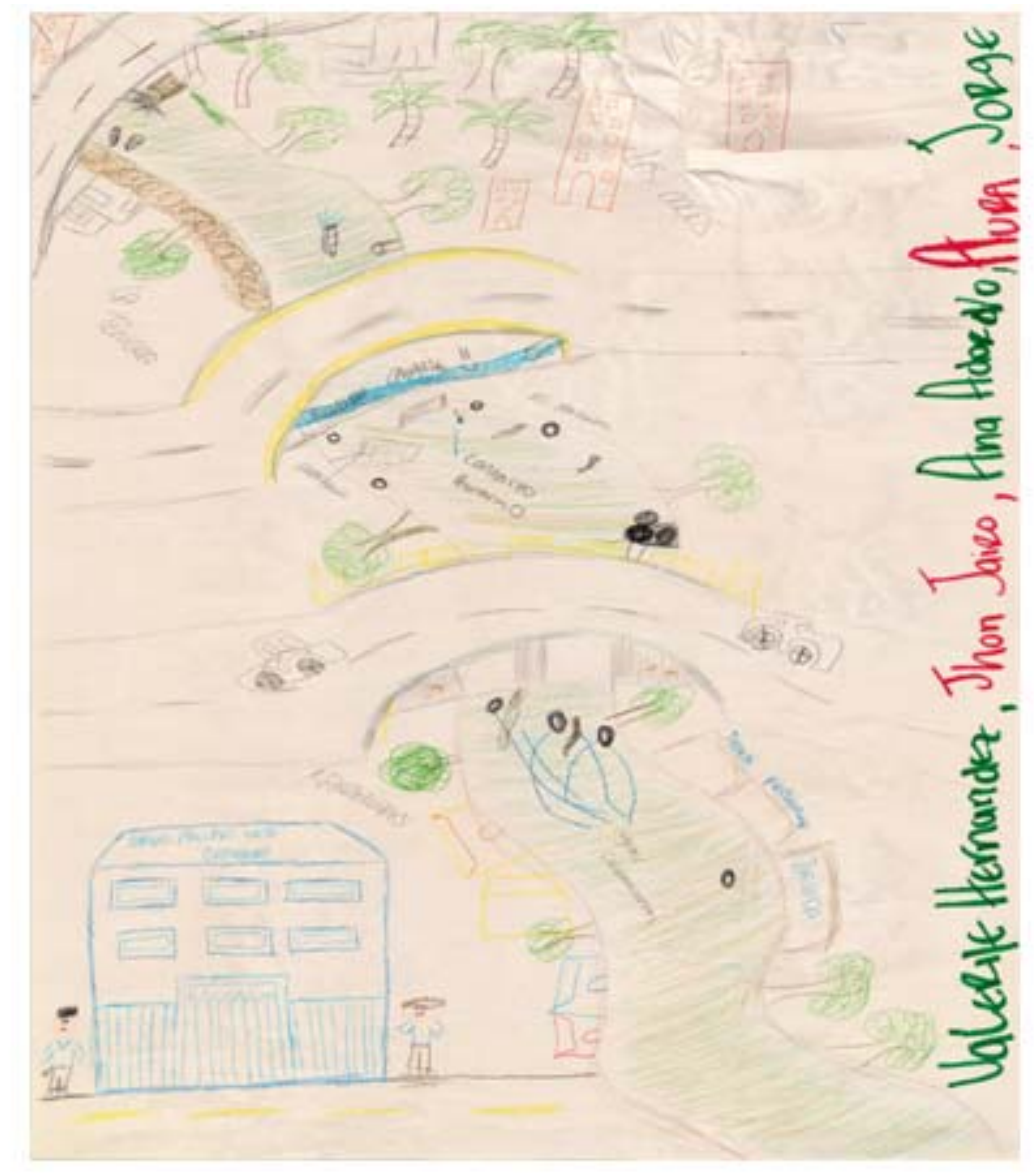

Figura 6. Imagen del taller de cartografía social, componente Suelo.

\section{Recurso Fauna}

De acuerdo con las observaciones de los participantes del taller, en la cuenca baja del río Manzanares aún se pueden ver animales como: sapos, iguanas, gusarapos, María mulata, renacuajos, garzas y perros callejeros. Antiguamente, se veían otros animales en abundancia tales como: peces, ardillas y un mayor número de los mencionados anteriormente. Esto nos muestra que la fauna ha disminuido con el tiempo, principalmente por el aumento de aguas negras y de contaminación en el río. Esta disminución se ha generado por causa de la contaminación, pues gracias a esto los animales han perdido fuentes de alimento, pues al perder otras especies animales y vegetales que los proveen de alimento, hay un impacto en las cadenas alimentarias de los ecosistemas, tanto terrestres como acuáticos, lo cual no da garantía de un hábitat adecuando, haciendo que los animales migren a lugares aptos o sencillamente mueran por escasez de recursos. Todo esto se ha impulsado gracias a la ausencia de un sentido de pertenencia con el río y a la falta de respeto con los animales en la zona. 
Para enfrentar esta situación, hay que cuidar a los animales y no contaminar su territorio, porque esto aumenta la mortalidad y se pueden extinguir de la zona. Esta idea se ve reforzada por parte de varios participantes, quienes afirman que: "El futuro de la fauna depende de cómo se hagan las cosas, si se limpia el río puede quedar agradable como hace años, pero si seguimos igual podría desaparecer el río". Esta idea, nos muestra la importancia de procesos de gestión que aseguren por ejemplo la descontaminación y recuperación del río, tales como la disposición de las aguas negras en una planta de tratamiento de aguas residuales -PTAR-; pero también, nos plantea la importancia del compromiso de cada ciudadano con el río, lo cual implica un proceso de transformación cultural.

En la Figura 7 se expresa lo observado en el recorrido, acá se muestra nuevamente la disminución de fauna en la cuenca baja, esto se contrasta con el desarrollo urbano de Santa Marta, a través del cual se ha generado presión sobre la fauna silvestre de la zona. Adicionalmente y en relación con el manejo de residuos, se resalta la presencia de animales como las ratas, lo cual además de tener un impacto cultural, genera un impacto negativo en la salud de los pobladores, pues como lo resaltan los participantes, son animales transmisores de enfermedades.

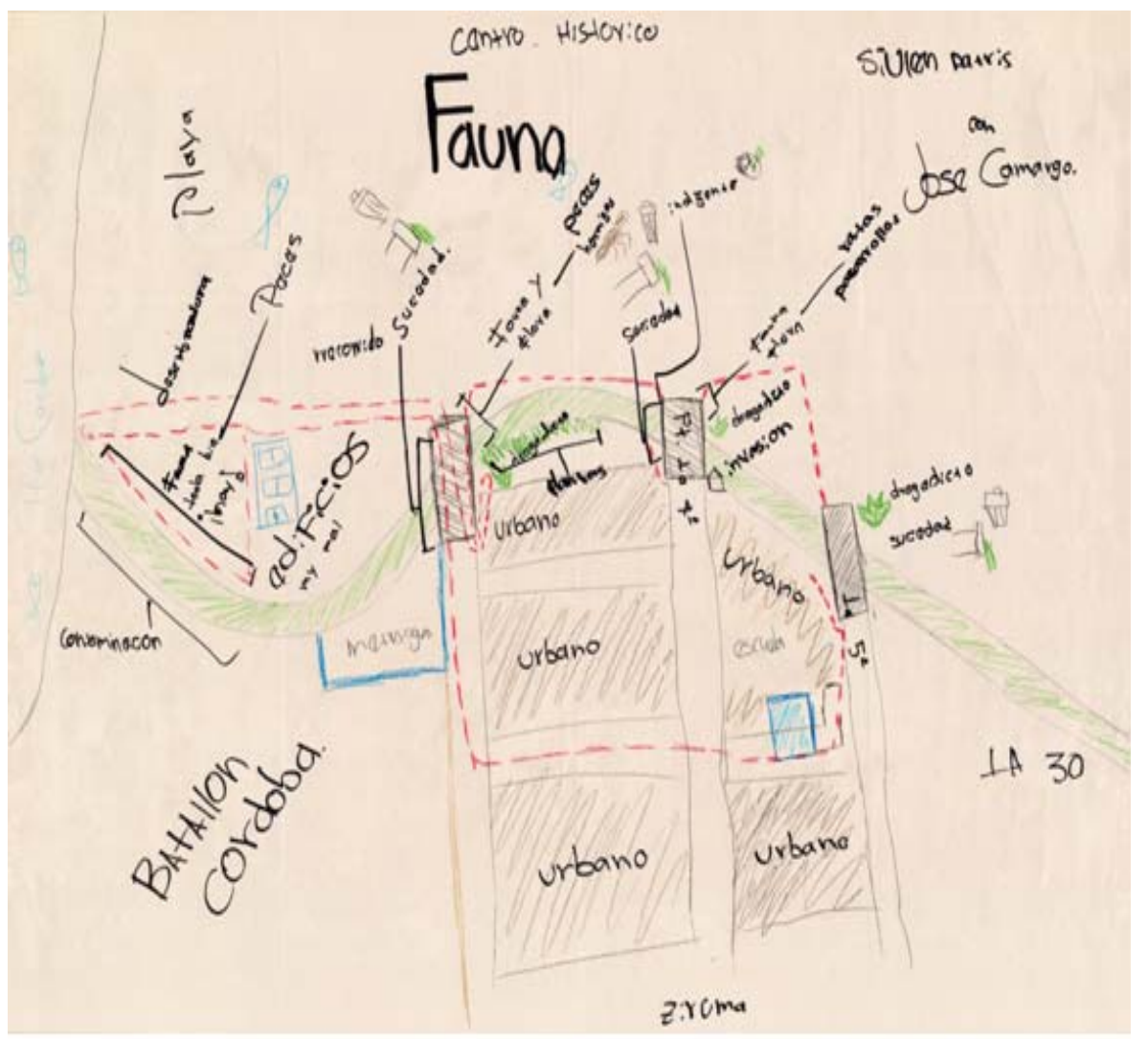

Figura 7. Imagen del taller de cartografía social, componente Fauna. 


\section{Recurso Flora}

Según los participantes del taller, las especies más importantes en el territorio son aquellas especies nativas, ya que son las que se adaptan mejor al territorio, fortalecen el suelo y evitan que la creciente del río pueda derrumbar parte del terreno.

Actualmente, solo se encuentra una cobertura vegetal abundante en zonas como el río Bonda y el Jardín Botánico de Santa Marta. Eso muestra la disminución de la cobertura vegetal, dada principalmente por el incremento de basuras, lo cual hace que escasee el agua limpia y que, además, haya estancamiento del agua por las basuras.

Los principales problemas percibidos por los participantes son la desaparición de plantas nativas, plantación de plantas no nativas, pérdida de resistencia del terreno por la tala de los árboles, deslizamiento de la tierra por la debilidad del terreno por falta de raíces que la sostenga. Frente a estos problemas se plantea como alternativa no plantar especies foráneas y detener la construcción de casas en la orilla del río. Se concluye, finalmente, que si el problema persiste nos encontraremos con pérdida total del recurso.

En la Figura 8 se muestra de manera gráfica lo expresado anteriormente; según los participantes existe un área con mayor concentración de áreas verdes que corresponde a la Quinta de San Pedro. Sin embargo, los participantes, no logran reconocer especies de flora de importancia en el territorio. 


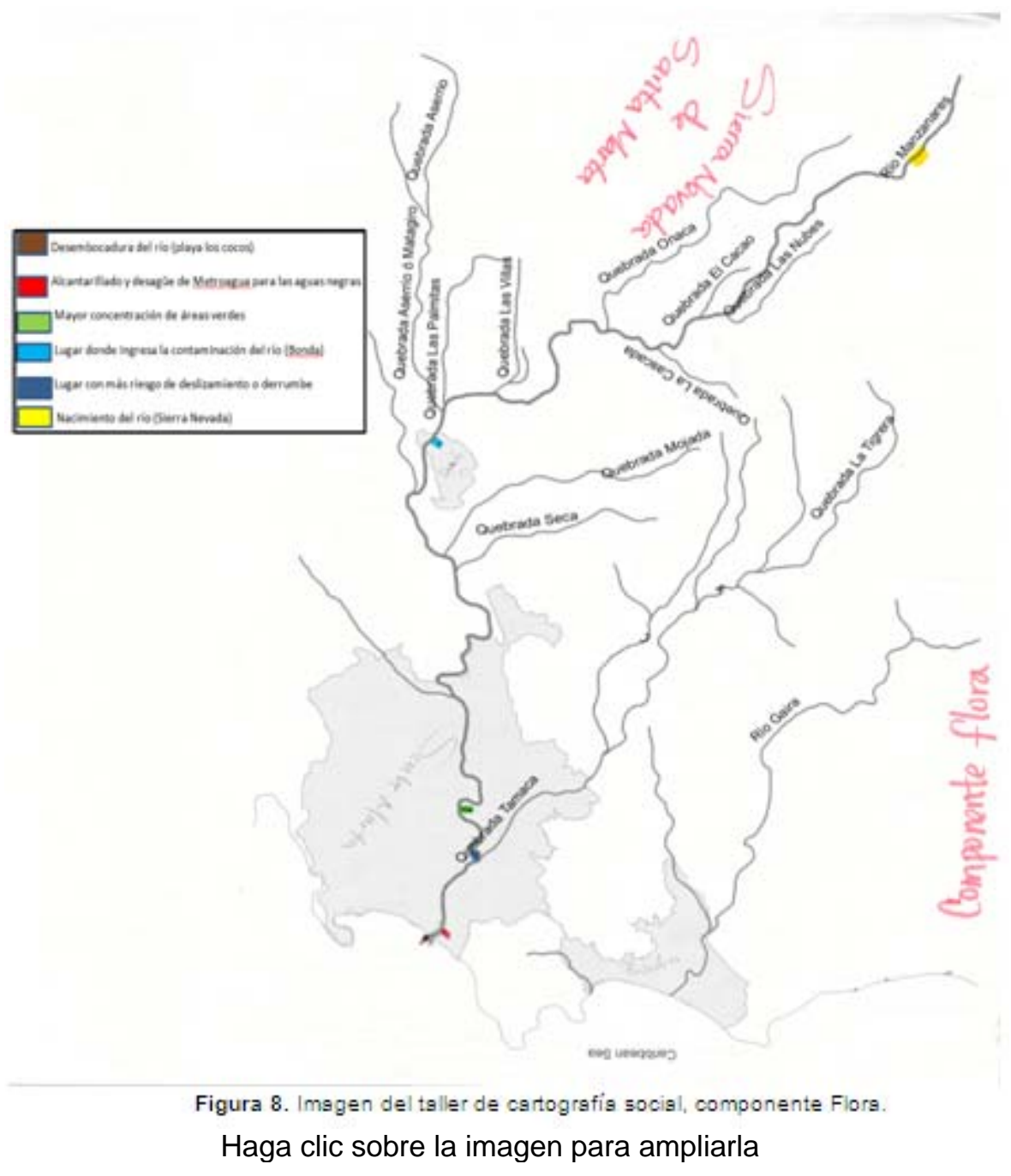

\section{Saneamiento Básico}

En la cuenca baja del río Manzanares se cuenta principalmente con los siguientes servicios públicos: agua potable, acueducto, energíaalumbrado, recolección de basura. Sin embargo en algunas zonas, especialmente en aquellas de invasión y construcción no planificada, no existe cobertura de varios de estos servicios.

Se afirma que no se hace un uso adecuado de los servicios presentes, ya que no se respeta el servicio de recolección de basuras, y esta es arrojada a las calles, la cual termina en las corrientes de agua que conducen al río. Asimismo, se observa que en muchas casas el uso del acueducto es inapropiado, porque algunos vertederos van directo al río.

Los principales problemas relacionados con el saneamiento básico son el uso de vertederos de agua directos al río, y el uso de este como basurero público. Estos son generados principalmente por las invasiones y los vertimientos directos. Esto genera como resultado inundaciones, malos olores, pérdida de cobertura vegetal, focos de infección. Algunas de las soluciones planteadas frente a estos problemas son la recuperación del cauce natural del río, el acondicionamiento de las zonas de descanso de este, y la restauración de la fauna y flora en la cuenca del río Manzanares. 
Se afirma que, partiendo de programas de sensibilización y concientización, se podría mejorar el estado actual del río, pero si no se genera un gran cambio, que parta también de la voluntad de entes gubernamentales y empresas, tal como lo es Metroagua, la situación va a continuar siendo la misma o peor, ya que el modelo de desarrollo urbanístico en Colombia y la ausencia de políticas que incentiven el trabajo en el campo, generarán una expansión en el caso urbano de Santa Marta y, por ende, un incremento en sus habitantes.

En la Figura 9 encontramos que los principales problemas percibidos en el recorrido, son la falta de alcantarillado en algunos barrios ubicados sobre la ronda hídrica del río, contaminación por residuos sólidos, vertimiento de aguas negras y las invasiones que a su vez generan problemas de inseguridad.

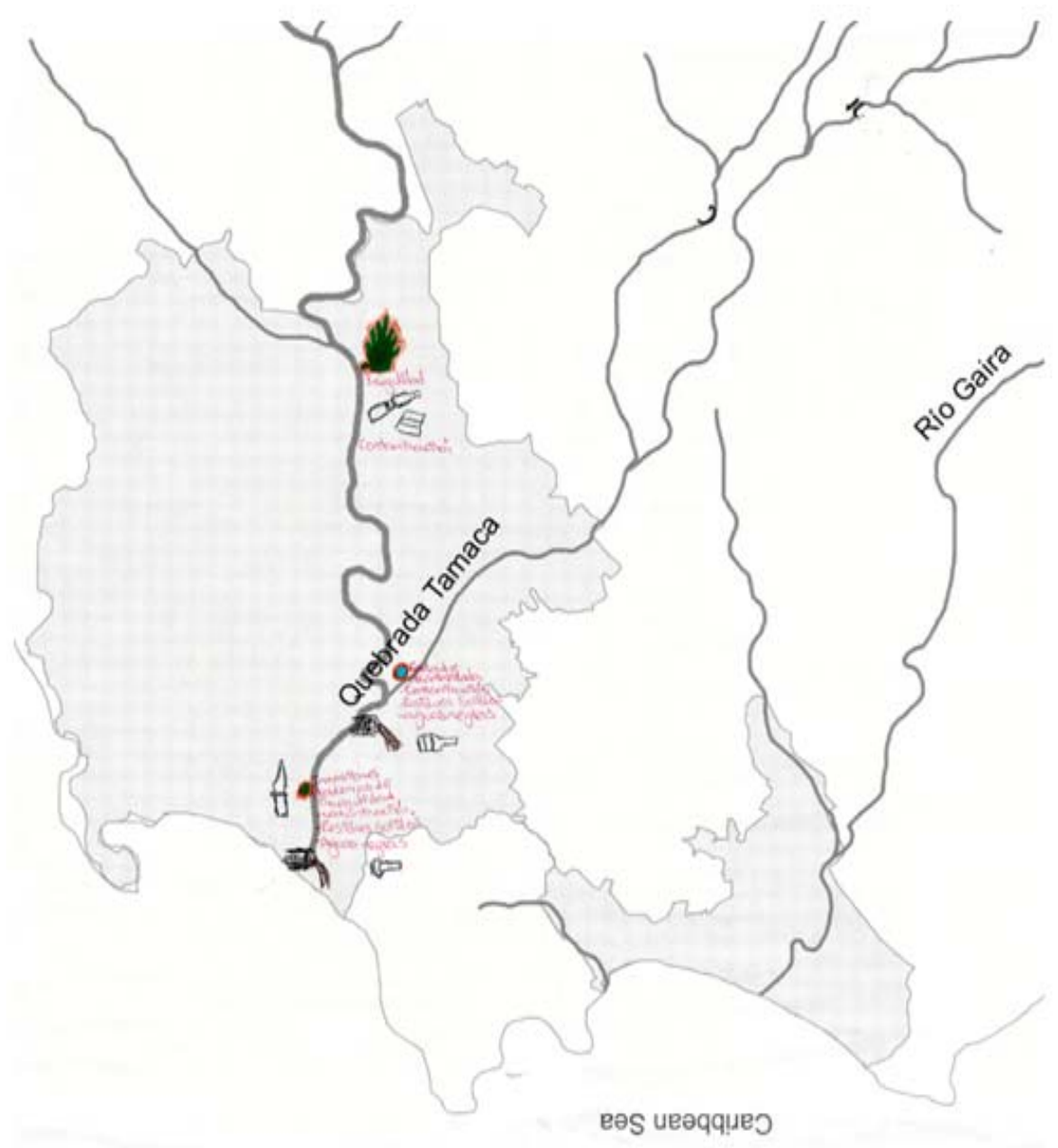

Figura 9. Imagen del taller de cartografía social, componente Saneamiento Básico.

\section{Percepción de familias asentadas en la cuenca baja del río Manzanares}

La aproximación a la percepción de la comunidad se recopiló a través de una encuesta aplicada a 30 familias localizadas en la cuenca baja del río Manzanares dentro de la ciudad de Santa Marta. Gran parte de ellas viven 
en barrios afectados por las principales problemáticas del río, especialmente en el barrio Manzanares y en La Tenería.

\section{Aporte de aguas residuales}

Es importante resaltar que gran parte de los adultos encuestados resaltan como problemática principal la falta de conciencia ciudadana, ausencia de una cultura y el poco sentido de pertenencia por parte de la comunidad y, en especial, por los jóvenes que habitan la ciudad.

Sin embargo, la mayoría de las personas encuestadas, asegura que el mayor problema del río se genera a causa del vertimiento de aguas negras sin ningún tratamiento realizado por la empresa Metroagua; esta es la empresa encargada de prestar los servicios de acueducto y alcantarillado en el Distrito Turístico, Cultural e Histórico de Santa Marta. Adicionalmente, se asegura que el aporte de esta empresa se hace de forma diaria, principalmente en los barrios de La Tenería y La Platina. Este aporte genera malos olores en la zona y, adicionalmente, un aumento en la contaminación del agua del río, lo cual a su vez ha llevado a desencadenar problemas de salud como enfermedades estomacales y otras causadas por la proliferación de mosquitos.

Sin embargo, algunos aseguran que el problema del vertimiento de aguas negras no se da solo por parte de la empresa Metroagua, sino también por el Batallón y lavaderos de carros, los cuales se encuentran ubicados en la cuenca del río Manzanares, muy cerca del él, generando a su vez aportes, pero en menor medida.

\section{Manejo de residuos sólidos}

De acuerdo con el orden de importancia para la comunidad, encontramos que el siguiente problema encontrado es el aporte de residuos sólidos sin control; este genera una acumulación de basura en ciertas partes del río, especialmente en sus orillas, lo cual se relaciona directamente con el aumento de la contaminación del agua.

Para cierta parte de la comunidad, el problema de las basuras está ligado a otro gran problema y es la invasión de la ronda hídrica y la construcción de viviendas de manera no planificada, la cual incrementa el aporte de residuos al río y en general a las zonas adyacentes a este.

\section{Otros factores}

Al mismo tiempo, se resalta que una causa importante para el deterioro del río ha sido la falta de voluntad política de los dirigentes, la inoperancia de las autoridades ambientales, el olvido del Distrito y la poca regulación hídrica que existe en la región. Adicionalmente, se citan otros problemas como la extracción de arena del río sin regulaciones, deforestación y sedimentación.

\section{Acciones frente a las problemáticas desde la comunidad local}

Los principales mecanismos para hacer frente a las problemáticas citadas, que reconocen los pobladores de la cuenca baja, son principalmente los medios que han utilizado para solicitar el cierre de las tuberías de Metroagua que generan una gran contaminación hídrica. Por tal razón, la mayoría de las acciones que la comunidad menciona hacen referencia a 
cartas de manifiesto, tutelas, comunicados de las juntas de acción comunal y otras herramientas comunitarias para solicitar de manera formal el cierre de estas tuberías.

En contraste, muy pocos reconocen acciones que puedan hacer personalmente para proteger el río, algunos incluso aceptan que no hacen nada para prevenir y enfrentar los problemas. Solo algunos reconocen que la principal acción con la cual aportan es evitando arrojar basuras al río. Se encontraron dos casos específicos en los cuales la comunidad se ha involucrado directamente con otro tipo de acciones: uno de los miembros de la comunidad afirma que desde su labor educativa ha tratado de orientar a estudiantes hacia la resolución de los problemas locales como es el caso del río. Por otra parte, un adulto mayor afirma que ha apoyado la iniciativa de la Fundación para la Investigación Atlántida Marina-Fiatmar de recuperación del ecosistema de manglar en la cuenca baja.

\section{Acciones potenciales para la protección del río}

A pesar de que para la mayoría de personas abordadas a través del proyecto, el principal problema es el aporte de aguas de Metroagua, solo cuatro personas afirman que una acción importante es quitar el desagüe de esta empresa y trabajar de la mano de ellos para mejorar esta situación. Otros expresan que en general hay que quitar todo tipo de desagüe que aporte aguas negras al río.

Por otra parte, se resalta que las acciones deben enfocarse principalmente en el desarrollo de campañas educativas y de cultura ciudadana, dirigidas a concientizar y sensibilizar a los habitantes de Santa Marta frente a su responsabilidad en la protección del río; lo cual mejorará y cambiará los hábitos de los habitantes de la ciudad. Adicionalmente, se menciona que es importante llevar a cabo capacitaciones a miembros de la comunidad que puedan replicar el mensaje a otras personas.

Otro aspecto que resaltan las personas encuestadas y al que le dan gran importancia, es el manejo de los residuos sólidos. Gran parte de ellas, expresan que es fundamental que tanto la comunidad aledaña al río como cualquier habitante o visitante de la ciudad no arroje residuos sólidos a este; esto disminuiría en gran parte los problemas que esta fuente hídrica afronta.

Otros dos temas relevantes para la comunidad son: 1) Recuperación de la ronda hídrica a partir de la reubicación de familias que viven sobre esta y 2) fortalecimiento de los mecanismos de control y vigilancia por parte de las autoridades ambientales pertinentes con el fin de disminuir y controlar los vertimientos de aguas negras y residuos sólidos. Se expresa que estos mecanismos de control deben estar acompañados de multas y sanciones a quienes generen este tipo de impactos, todo bajo el supuesto de que quien contamina paga.

Finalmente, algunos proponen que se deben hacer estudios sobre el estado actual del río para tener una línea base a partir de la cual se puedan generar alternativas de recuperación.

Se consideran otras alternativas como: recuperar las condiciones ecológicas de la ronda del río, canalización del río, Plan de manejo del río, recuperación de zonas verdes, quitar la base Ejército, limpieza general, alcantarillado en Puerto Mosquito, entre otras. 
A continuación, algunas iniciativas de jóvenes de la zona, quienes plantean en sus propias palabras: ¿Cómo desde su papel en la sociedad pueden aportar a la recuperación del río?

Frente a esta pregunta, se generaron diversas respuestas resumidas en las siguientes citas:

- "Proteger, cuidar especies nativas".

- "Recoger la basura y no cazar los animales, reforestar, limpiar".

- "Dejar de tirar basura para que no se siga contaminando el río, cultivar más plantas y árboles".

- "Proteger y Restaurar cuencas."

- "Diseñar proyectos de limpieza de la cuenca del río Manzanares, limpieza, capacitación y protección".

- "Diseñar proyectos ambientales de protección de la flora y fauna característica de la cuenca del Manzanares; reforestación, descontaminación".

- "Cultivo de árboles, limpiezas, reciclaje, valoración".

- "Concientizar y tener iniciativa".

- "Podemos ayudar a concientizar a los habitantes cercanos al río sobre la importancia y cuidado del río Manzanares además de la recuperación de las riberas del río; cuidado y recuperación".

\section{Influencia del estado del río sobre los habitantes de la ciudad}

Al abordar un problema ambiental desde el saber local es necesario no solo analizar cuál es el problema, sino también entender cuál es la forma en que las comunidades se ven afectadas directa o indirectamente por este. Para generar este conocimiento se cuestionó a la comunidad a cerca de los efectos de los problemas del río en ellos y en sus familias. A continuación se listan algunos factores mencionados:

- "Los problemas ambientales, las inundaciones, el estado del río provoca proliferación de vectores y roedores plaga derivando en enfermedades. $\mathrm{Da}$ un mal aspecto a la ciudad, provoca incluso daños a su estructura".

- "A los ciudadanos en general se les ha quitado el privilegio de tener un río sano para el disfrute sano como se hacía hace ya muchos años cuando nos bañábamos en él y era algo muy apreciado".

- "Mal aspecto de la ciudad, daños durante las inundaciones, malos olores, enfermedades en algunas zonas".

- "La contaminación afecta la bahía de Santa Marta, lo que afecta la salud, el turismo en la zona costera y se ha quitado la posibilidad de tener un río atractivo para los samarios y visitantes".

- "El desplazamiento de personas los obliga a mudarse cerca al río y estas viviendas obstruyen el espacio del río y su flujo natural y eso provoca las inundaciones".

- "Mal aspecto de la ciudad, inseguridad en los sitios del río donde acampan los indigentes, delincuentes y adictos".

- "Inundaciones provocan desastres en la ciudad, la contaminación de las aguas es peligrosa para la salud".

- "Disminución de recurso agua, Inundaciones en la ciudad provocan caos".

- "Mal aspecto de la ciudad, inundaciones y caos vehicular y algunas enfermedades derivadas del estado del río".

- "Inundaciones, daños en la ciudad, reboce de aguas negras y mal aspecto de la ciudad". 
- "Mal aspecto en muchos lugares de Santa Marta ya que el río es visto como una cloaca que recorre la ciudad y la siguen tratando como tal. Las inundaciones y represamientos también generan situaciones incómodas como malos olores y mosquitos".

- "En el barrio el suelo cede frecuentemente y las casas se resquebrajan o fracturan y eso es un riesgo para las personas".

- "Malos olores, mal aspecto del río, estancamiento de aguas, problemas respiratorios".

- "Mal olor a las 6, ratas, erosión del patio, proliferación de mosquitos, enfermedades de piel, diarrea, etc.".

Como se observó anteriormente, uno de los problemas que se resalta con cierta frecuencia es el de los malos olores; por tal razón, se preguntó directamente a los pobladores si este problema se presentaba en sus zonas de vivienda. El $53 \%$ de las familias encuestadas afirma sufrir de malos olores en sus zonas y el $47 \%$ restante dice que no. Esto se da ya que la encuesta abordó diferentes barrios a orilla del río, en los que la problemática en mención se puede percibir en diferentes proporciones. La mayor parte de personas que afirman sufrir de malos olores viven en el barrio La Tenería ubicado cerca de la Calle 29 con Carrera 1.

Adicionalmente, se indagó si es adecuado bañarse en el río o si por el contrario pueden tener problemas de salud. Solo el $3 \%$ de los encuestados afirman que pueden bañarse en el río sin correr ningún tipo de riesgo, pero el resto de familias, es decir el $97 \%$ de los encuestados, opinan que no pueden bañarse en el río dados los riesgos de salud que pueden tener.

Adicionalmente, solo el 3\% opina que el agua del río puede ser usada para el consumo en el hogar; las otras personas, por el contrario, creen que esta agua no debe usarse para fines domésticos.

Esto confirma la percepción que la comunidad de la cuenca baja tiene del río, pues vemos que la gran mayoría de las personas abordadas a través de la encuesta y los talleres participativos, está de acuerdo con que la principal problemática es la contaminación del agua, lo cual implica que los servicios ecosistémicos que este río prestaba en su cuenca baja, se hayan perdido en su mayoría y otros se hayan deteriorado en gran medida.

\section{CONCLUSIONES Y TRABAJO FUTURO}

El principal problema percibido por la comunidad en la cuenca baja del río Manzanares, es la contaminación del agua del río, generada principalmente por el vertimiento de aguas residuales y el aporte no controlado de residuos sólidos. Según la comunidad, esto se genera a su vez por la falta de una cultura ambiental en los pobladores de la ciudad.

El recorrido de reconocimiento ambiental y territorial, permitió evidenciar que a pesar de que la mayoría de los participantes viven en el área de influencia, muy pocos habían visto de cerca el río y sus principales problemas asociados a cada uno de los recursos que se tuvieron en cuenta durante la actividad. 
Como trabajo futuro, es necesario el desarrollo de Proyectos Ambientales Escolares -PRAES-, los cuales son proyectos institucionales en la educación básica y secundaria, que se convierten en estrategia para la implementación de la Política Nacional de Educación Ambiental; se pretende generarlos en colaboración con las autoridades ambientales, de manera que en estos proyectos se realicen con determinada periodicidad recorridos semejantes al reportado en este trabajo.

Adicionalmente, es necesario el apoyo a la implementación de Proyectos Ambientales Comunitarios -PROCEDAS-, los cuales también se establecen como herramienta de implementación de la Política Nacional de Educación Ambiental, con miras al fortalecimiento de comunidades locales frente a situaciones ambientales. Estos a su vez podrán guiar a la comunidad en procesos de empoderamiento de su territorio, con el fin de generar una mayor conciencia ambiental e impulsar un proceso de transformación cultural.

\section{AGRADECIMIENTOS}

Los autores quieren agradecer, en primera instancia, a la Universidad Sergio Arboleda por su apoyo en el desarrollo del presente proyecto. Adicionalmente, a todas las personas de Santa Marta que fueron partícipes de las actividades del trabajo. De igual manera, a las instituciones que acompañaron el proceso, principalmente a ESPA.

\section{POTENCIAL CONFLICTO DE INTERESES}

Los autores declaran que por la realización de este trabajo no existe ningún conflicto de intereses.

\section{FUENTES DE FINANCIACIÓN}

La principal fuente de financiación de este proyecto es la Universidad Sergio Arboleda a través de la Dirección de Investigación e Innovación de la misma.

\section{REFERENCIAS}

- CHÁVEZ, N. (2001). La Cartografía social: un procedimiento para la planeación participativa en el nivel local. Santiago de Cali, Valle del Cauca: Corporación Autónoma Regional del valle del Cauca.

- Delgado, S., Trujillo, J. y Torres, M. H. (2013). La huella hídrica como una estrategia de educación ambiental enfocada a la gestión del recurso hídrico: ejercicio con comunidades rurales de Villavicencio. Revista Luna Azul, 36. Recuperado 
de http://lunazul.ucaldas.edu.co/index.php?option=content\&task=view\&id= $\underline{794}$

- $\quad$ Dye, C. (2008). Health and urban living. Science, 319, 766-769.

- Gómez-Baggethun, E. y Barton, D. N. (2013). Classifying and valuing ecosystem services for urban planning. Ecological Economics, 86, 235245.

- Gómez, M., Gutiérrez, I. y Montes, F. (2013). Percepción local acerca de la calidad del agua y la herpetofauna en fincas ganaderas del municipio de Matiaguas, Nicaragua. Revista Luna Azul, 38. Recuperado de http://lunazul.ucaldas.edu.co/index.php?option=content\&task=view\&id= $\underline{893}$

- Lee, K. N. (2006). Urban sustainability and the limits of classical environmentalism. Environ. Urban, 18, 9-22.

- Mora, H. y Jaramillo, C. (2003). Aproximación a la construcción de cartografía social a través de la geomática. Ventana Informática, 11, 129146. Recuperado de http://www.academia.edu/10937788/CENTRO DE INVESTIGACIONES Y DESARROLLO FACULTAD DE INGENIER\%C3\%8DA APROXIMACI $\%$ C3\%93N A LA CONSTRUCCI\%C3\%93N DE CARTOGRAF\%C3\%8 DA SOCIAL A TRAV\%C3\%89S DE LA GEOM\%C3\%81TICA H\%C3\% 89CTOR MORA-P\%C3\%81EZ

- Patiño, M. y Tobasura, I. (2011). Tomadores de decisión en sistemas ganaderos de la cuenca alta del río Guarinó (Caldas, Colombia): percepción de problemas ambientales y prácticas de conservación del agua. Revista Luna Azul, 33. Recuperado de http://lunazul.ucaldas.edu.co/index.php?option=content\&task=view\&id=653

- Tréllez Solis, E. (2002). La educación ambiental comunitaria y la retrospectiva: una alianza de futuro. Tópicos en Educación Ambiental, 4(10), 7-21.

- United Nations (2010). World Urbanization Prospects: The 2009 Revision. UN Department of Economic and Social Affairs, Population Division, New York. Recuperado de http://esa.un.org/wup2009/unup/index.asp?panel

- Vargas, C., Briones, C. D., Mancha, M. del P. S., Músquiz, P. G. y Vargas, A. (2013). Conciencia ambiental de los habitantes de la colonia Emilio Portes Gil en la H. Matamoros, Tamaulipas. Revista Luna Azul, 37. Recuperado de http://www.redalyc.org/pdf/3217/321729206011.pdf

- Vollmer, D. y Grêt-Regamey, A. (2013). Rivers as municipal infrastructure: Demand for environmental services in informal settlements along an Indonesian river. Global Environmental Change, 23, 1542-1555.

- Wang, X., Li, J., Li, Y., Shen, Z., Wang, X., Yang, Z. y Lou, I. (2014). Is urban development an urban river killer? A case study of Yongding Diversion Channel in Beijing, China. Journal of Environmental Sciences, 26, 1232-1237.

1. Máster en Ciencias Ambientales. Grupo de Investigación IDEASAMedio Ambiente y Sostenibilidad, Universidad Sergio Arboleda. Bogotá, Colombia. angela.plata@usa.edu.co

2. Máster en Gestión y Evaluación Ambiental. Grupo de Investigación IDEASA-Medio Ambiente y Sostenibilidad, Universidad Sergio Arboleda. Bogotá, Colombia.danny.ibarra@usa.edu.co 
Para citar este artículo: Plata, Á. M. e Ibarra Vega, D. (2016). Percepción local del estado ambiental en la cuenca baja del río Manzanares. Revista Luna Azul, 42, 235-255. Recuperado de http://200.21.104.25/lunazul/index.php?option=com_content\&view=artic le\&id $=137$ 